\title{
Restoration of sensory dysfunction following peripheral nerve injury by the polysaccharide from culinary and medicinal mushroom, Hericium erinaceus (Bull.: Fr.) Pers. through its neuroregenerative action
}

\author{
Kah-Hui WONG ${ }^{1,2 *}$, Gowri KANAGASABAPATHY ${ }^{2,3}$, Robiah BAKAR ${ }^{1}$, Chia-Wei PHAN ${ }^{2,4}$, \\ Vikineswary SABARATNAM ${ }^{2,3}$
}

\begin{abstract}
Peripheral nerves have the unique capability to regenerate after injury. Insights into regeneration of peripheral nerves after injury may have implications for neurodegenerative diseases of the nervous system. We investigated the ability of polysaccharide from Hericium erinaceus mushroom in the treatment of nerve injury following peroneal nerve crush in Sprague-Dawley rats by daily oral administration. In sensory functional recovery test, the time taken for the rats to withdraw its hind limb from contact with the hot plate was measured. The test revealed acceleration of sensory recovery in the polysaccharide group compared to negative controls. Further, peripheral nerve injury leads to changes at the remotely located DRG containing cell bodies of sensory neurons. Immunofluorescence studies showed that Akt and p38 MAPK were expressed in DRG and strongly upregulated in polysaccharide group after peripheral nerve injury. The intensity of endothelial cells antigen-1 that recognized endothelial cells in the blood vessels of distal segments in crushed nerves was significantly higher in the treated groups than in the negative control group. Our findings suggest that $H$. erinaceus is capable of accelerating sensory functional recovery after peripheral nerve injury and the effect involves the activation of protein kinase signaling pathways and restoration of blood-nerve barrier.
\end{abstract}

Keywords: Hericium erinaceus; polysaccharide; peripheral nerve regeneration; sensory functional recovery; protein kinases; blood-nerve barrier.

Practical Application: Hericium erinaceus enhanced nerve regeneration and accelerated functional recovery after crush injury. Patients who receive $H$. erinaceus may experience a more expeditious improvement in the quality of life after injury. It is noted that the neurotherapeutic effects of the polysaccharide was comparable to those elicited by mecobalamin, a drug used in peripheral nerve disorders. However, taking mecobalamin for the treatment of nerve injury gives rise to side effects such as gastrointestinal and dermatological problems

\section{Introduction}

Medicinal properties of Hericium erinaceus (Bull.: Fr) Pers., also known as Lion's Mane, Monkey's Head and Yamabushitake have been well known for hundreds of years in traditional Chinese and Japanese herbal medicine to treat various human diseases. The most promising activity of $H$. erinaceus is the stimulation of nerve growth factor (NGF) synthesis by hericenones from fruit bodies and erinacines from mycelium (Kawagishi et al., 2008). Extract of fruit bodies exerted neurotrophic action and improved myelination process in mature fibers of neuronal cells in vitro (Moldavan et al., 2007), and promoted normal development of cultivated cerebellar cells as well as regulatory effect on the process of myelin genesis in vitro (Kolotushkina et al., 2003).

The mushroom has been extensively tested in in vitro trials as the neurite outgrowth stimulator in the cultured cells of neural hybrid clone NG108-15 and rat phechromocytoma PC12 in our Fungal Biotechnology laboratory, Mushroom Research Centre, Faculty of Science (Wong et al., 2007; Lai et al., 2013;
Phan et al., 2014). Phan et al. (2014) isolated four benzyl alcohol derivatives namely hericenones $\mathrm{B}-\mathrm{E}$ from the fruit bodies of $\mathrm{H}$. erinaceus cultivated in tropical conditions in Malaysia. Hericenone E exerts neurotrophic activity in PC12 cells, and this activity is mediated by extracellular signal-regulated kinase 1/2 (ERK1/2) and phosphoinositide-3-kinase/protein kinase B (PI3K/Akt) signaling pathways. The bioactive components in fruit bodies of $H$. erinaceus could interact with NGF to enhance neurite outgrowth by upregulating common pathways linked to the induction of NGF signaling, namely TrkA-associated tyrosine kinase that leads to sustained activation of ERK and PI3K/Akt that is crucial for neurite outgrowth.

Peripheral nerve injury is a serious health concern for society, which always results in restricted activity or life-long disability. Traffic crashes induce traumatic nerve injury resulting in the disruption of intraneural circulation. Although microsurgical treatments for nerve injuries have been improved over the past decades, the outcome of peripheral nerve injury repair remains 
unsatisfactory. Peripheral nervous system has an intrinsic ability for repair and regeneration, therefore making it a well-accepted neuroregeneration research model.

From the established perspective, we explored the ability of locally cultivated $H$. erinaceus in the enhancement of peripheral nerve regeneration and acceleration of motor functional recovery after crush injury. Upregulation of signaling pathways as well as axonal protein synthesis and degradation are consistent with the beginning of motor functional recovery of injured limb (Wong et al., 2009a, 2011, 2012, 2014). It has been shown that cultivation conditions did not affect selected bioactive properties of H. erinaceus grown in tropical Malaysia (Wong et al., 2009b).

The specific aims of the study were to investigate the neuroregenerative properties of polysaccharide from locally cultivated $H$. erinaceus on sensory functional recovery following crush injury to the peroneal nerve by behavioral experiment as assessed by hot plate test, expression of protein kinase B (Akt) and p38 mitogen-activated protein kinase (p38 MAPK) in the dorsal root ganglia (DRG), and restoration of blood-nerve barrier (BNB) by immunofluorescence analysis.

\section{Materials and methods}

\subsection{Mushroom and peparation of polysaccharide}

Fresh fruit bodies of $H$. erinaceus were obtained from Vita Agrotech mushroom farm in Tanjung Sepat, Selangor, Malaysia. Preparation of hot water polysaccharide was carried out according to the method of Roy et al. (2008). $500 \mathrm{~g}$ of fruit bodies were boiled in $500 \mathrm{~mL}$ of distilled water for $8 \mathrm{~h}$. The whole mixture was kept overnight at $4{ }^{\circ} \mathrm{C}$ and then filtered through linen cloth. The filtrate was centrifuged at $8000 \mathrm{rpm}$ for $45 \mathrm{~min}$ at $4{ }^{\circ} \mathrm{C}$. Supernatant was collected and precipitated in ethanol at $1: 5(\mathrm{v} / \mathrm{v})$. It was kept overnight at $4^{\circ} \mathrm{C}$ and again centrifuged. The precipitated material was washed with ethanol four times and then freeze-dried. The freeze-dried material was dissolved in $30 \mathrm{~mL}$ of distilled water and dialyzed through dialysis tubing cellulose membrane (Sigma-Aldrich, St. Louis, MO, USA, retaining more than MW 12400) against distilled water for $4 \mathrm{~h}$ to remove low molecular weight materials. The aqueous solution was then collected from the dialysis bag and freeze-dried to yield polysaccharide of $1.4 \mathrm{~g}$.

\subsection{Principle of animal grouping}

The rat experiment was approved by the Institutional Animal Care and Use Committee, Faculty of Medicine, University of Malaya, Ethics Ref No 2013-10-08/ANAT/R/WKH. Eighteen adult female Sprague-Dawley rats were randomly assigned into three groups of six rats each. Negative control group received daily oral administration of distilled water $(10 \mathrm{~mL} / \mathrm{kg}$ body weight per day), positive control group received mecobalamin (Natural Factors $^{\circledast}$ batch $020564,0.013 \mathrm{mg} / \mathrm{mL} / \mathrm{kg}$ body weight/day) and experimental group received polysaccharide of $H$. erinaceus (30 $\mathrm{mg} / \mathrm{mL} / \mathrm{kg}$ body weight/day) for 14 days to function as pre-treatment before injury.

\subsection{Surgical procedure}

After 14 days of pre-treatment, the rats were anesthetized with an intraperitoneal injection of $3.5 \%$ chloral hydrate (10 mL/kg body weight), then shaved and washed with antiseptic solution before positioning for surgery. The right sciatic nerve and its two major branches were exposed through a gluteal muscle-splitting incision. A crush injury was created using a fine watchmaker forceps no. 4 for 10 seconds on the peroneal nerve at $10 \mathrm{~mm}$ from extensor digitorum longus muscle and complete crush was confirmed by presence of a translucent band across the nerve. All operations were performed on right limb and the left limb served as an uninjured control. After closing the incision with sutures, veterinary wound powder was applied to wounds. Distilled water, mecobalamin or polysaccharide was continuingly fed for another 28 days. All rats were observed for general well-being and had ad libitum access to food and water throughout the study.

\subsection{Functional assessment of sensory recovery (temperature sensitivity assay)}

The hot plate test is commonly used for evaluating thermal pain sensitivity. During the experiment, the rats were introduced into an open-ended cylindrical space with a floor consisting of a heated plate (Panlab, S.L. Spain). The plate heated to $50{ }^{\circ} \mathrm{C}$ produces two behavioural components that can be measured in terms of their reaction times, namely paw licking and jumping (Figure 1). Both are considered to be supraspinally integrated responses. The time elapsed from the onset of hot plate contact to withdrawal of hind limb was measured by a built-in timer activated by an external foot switch and recorded as withdrawal reflex latency (WRL). According to $\mathrm{Hu}$ et al. (1997), normal rats withdraw their paws from the hot plate within 4.3 seconds or less. Jumping responses were obtained before injury (day 0 ) and on day $4,8,12,16,20,24$ and 28 after injury.

The affected right limbs were tested 3 times, with an interval of 2 min between consecutive tests to prevent sensitization and the 3 latencies were averaged to obtain a final result. If there was no withdrawal of hind limb after $12 \mathrm{~s}$ of stimulation, the heat stimulus was removed to prevent tissue damage, and the animal was assigned the maximal WRL of $12 \mathrm{~s}$ (Campbell, 2001).

\subsection{Immunofluorescence and microscopy}

The expressions of Akt and p38 MAPK in the DRG of normal and injured nerves, and RECA-1 in the normal and injured nerve were investigated after 12 days of crush injury. The selected time period for immunofluorescence analysis is associated with significantly lower WRLs of treated rats than the negative control group that marks the onset of regeneration. In the no-primary antibody control for the validation of the Akt, p38 MAPK or RECA-1 assay, sections were incubated with secondary antibody alone without primary antibody in all runs.

\section{Analysis of Akt and p38 MAPK in the DRG}

Rats were perfused transcardially with $4 \%$ paraformaldehyde in $0.1 \mathrm{M}$ phosphate buffer saline. DRG were traced from their respective peroneal nerves to the intervertebral foramen [vertebral level- fifth lumbar (L5] to third sacral (S3)] and the surrounding 


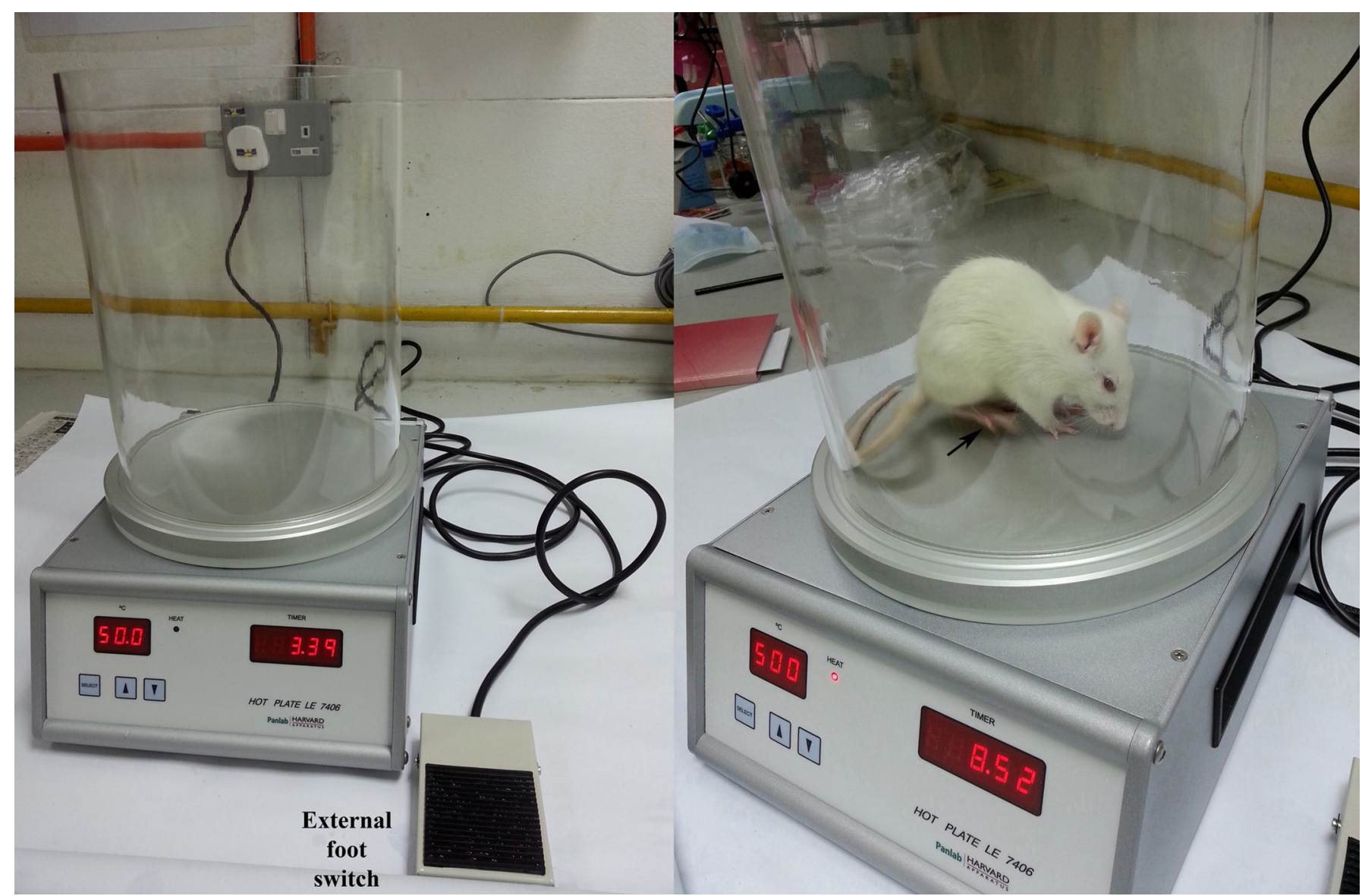

Figure 1. Hot plate apparatus comprises of an open-ended cylindrical space with a floor consisting of a heated plate. Skin of the plantar surface of the foot was stimulated by gently placing the rat on the heated surface. Thermal nociception was evaluated by observing the WRL of the right hind limb in response to heat stimulation (as indicated by an arrow) by a built-in timer activated by an external foot switch.

bone was removed with fine bone clippers before ganglia removal. L5 DRGs from crushed nerves (ipsilateral) and their uncrushed counterparts (contralateral) were dissected out. DRGs were fixed, processed and embedded in paraffin before sectioning.

Longitudinal sections ( $5 \mu \mathrm{m}$ ) of DRG were washed in $0.1 \mathrm{M}$ phosphate buffer saline containing $0.3 \%$ Triton X-100 (washing buffer). Non-specific binding sites were blocked with blocking buffer ( $10 \%$ normal sheep serum in washing buffer) for $1 \mathrm{~h}$ at room temperature. The sections were then incubated with a mixture of primary antibodies (rabbit polyclonal anti-Akt antibody, Cat. No. 9272, 1:100 dilution, Cell Signaling Technology, Beverly, MA or rabbit polyclonal anti-p38 MAPK, Cat. No. 09-272, 1:100 dilution, Millipore, Temecula, CA) and mouse monoclonal anti-neurofilament 200 (NF-200), a marker for myelinated A-fibres, Cat. No. N0142, 1:200 dilution, Sigma-Aldrich, St. Louis, $\mathrm{MO}$, in blocking buffer at $4{ }^{\circ} \mathrm{C}$ for $20 \mathrm{~h}$ in humidity chamber, washed with washing buffer and further reaction with a mixture of secondary antibodies (Cy3-conjugated sheep anti-rabbit IgG, Cat. No. C2306, 1:100 dilution and FITC-conjugated sheep anti-mouse IgG, Cat. No. F2266, 1:80 dilution, Sigma-Aldrich, St. Louis, MO, in blocking buffer) at room temperature for $1 \mathrm{~h}$. After the same washing procedure, sections were coverslipped with antifade reagent with 4,6-diamidino-2-phenylindole (DAPI).

\section{Analysis of RECA-1 in the peroneal nerve}

The right peroneal nerves were carefully dissected out and their proximal and distal ends were identified. The contralateral peroneal nerves were also obtained.

Longitudinal sections $(5 \mu \mathrm{m})$ of peroneal nerves were washed in washing buffer. Non-specific binding sites were blocked with blocking buffer (10\% normal rabbit serum in washing buffer) for $1 \mathrm{~h}$ at room temperature. The sections were then incubated with mouse monoclonal anti-rat endothelial cell antigen-1 (RECA-1) (Cat. No. MCA970R, 1:100 dilution, Serotec, UK) in blocking buffer at $4{ }^{\circ} \mathrm{C}$ for $20 \mathrm{~h}$ in a humidity chamber, washed with washing buffer and further reaction with FITC-conjugated rabbit anti-mouse IgG, Cat. No. F9137, 1:80 dilution, Sigma-Aldrich, St. Louis, MO, in blocking buffer at room temperature for $1 \mathrm{~h}$. After the same washing procedure, sections were coverslipped with DAPI.

\subsection{Image analysis}

Four sections of DRG or peroneal nerve were randomly selected from ipsilateral or contralateral side of each rat and tested for each antibody. Images were analyzed with Nikon Eclipse 80i upright microscope equipped with a digital color 
camera controller (DS-5Mc-U2), neutral density filter and NIS-Elements algorithm that enables maximum accuracy, resulting in ultra high-resolution images (NIS-Elements Advanced Research, Nikon, Japan). Intensity of Cy3 staining regions as an average figure that demonstrated immunoreactivities for Akt or p38 MAPK or FITC staining regions for RECA-1 was examined under fluorescence illumination.

\subsection{Statistical analysis}

The means of data ( $n=6$ animals per group) were subjected to a one-way analysis of variance (ANOVA) and the significance of the difference between means was determined by the Duncan's multiple range tests (DMRT) at 95\% least significant difference $(P<0.05)$. Data are expressed as mean \pm standard error.

\section{Results}

None of the rats in all groups were found to have skin lesions and signs of self-mutilation at any time throughout the experiment. Crush injury to the peroneal nerve results in foot drop. It can be defined as a significant weakness of ankle and toe dorsiflexion. The foot and ankle dorsiflexors include the tibialis anterior, extensor hallucis longus, and extensor digitorum longus. These muscles help the body clear the foot during swing phase and control plantar flexion of the foot on heel strike. The rats tend to drag the dorsum of their foot until reinnervation of axons into these muscles. The behaviours observed in rats subjected to the hot plate test fell into three main behavioral categories, namely exploratory (walking, sniffing), primary noxious-evoked (hind paw licking, front paw licking and shaking/stamping) and escape (climbing, jumping).

Figure 2 presents the data for the WRL during the healing period of 4 weeks. As expected, during the first week following peroneal nerve crush, rats were unable to respond to the hot stimulus. This indicates complete loss of thermal and nociceptive sensitivity at the sole of the right foot. Signs of recovery of foot's withdrawal response began at week 2 after crush injury. Thereafter, the WRL steadily improved during the 4-week recovery time. However, the normal value of 4 seconds was unachievable (Hu et al., 1997).

Negative control rats showed no signs of recovery of WRL response during the healing period of 4 weeks. The WRL at week 4 for the polysaccharide group was almost in the normal value range. The mean WRL of polysaccharide group was $5.6 \pm 0.4 \mathrm{~s}$. When the mean WRLs were compared at each time interval, the mean WRLs of the polysaccharide-treated rats were significantly less than the mecobalamin group from day 8 onwards $(P<0.05)$. Therefore, WRL in rats treated with polysaccharide recovered faster and better compared to those receiving mecobalamin from day 8 onwards $(P<0.05)$. There was no significant difference in mean WRLs between rats in mecobalamin and negative control groups after 4 and 8 days of injury.

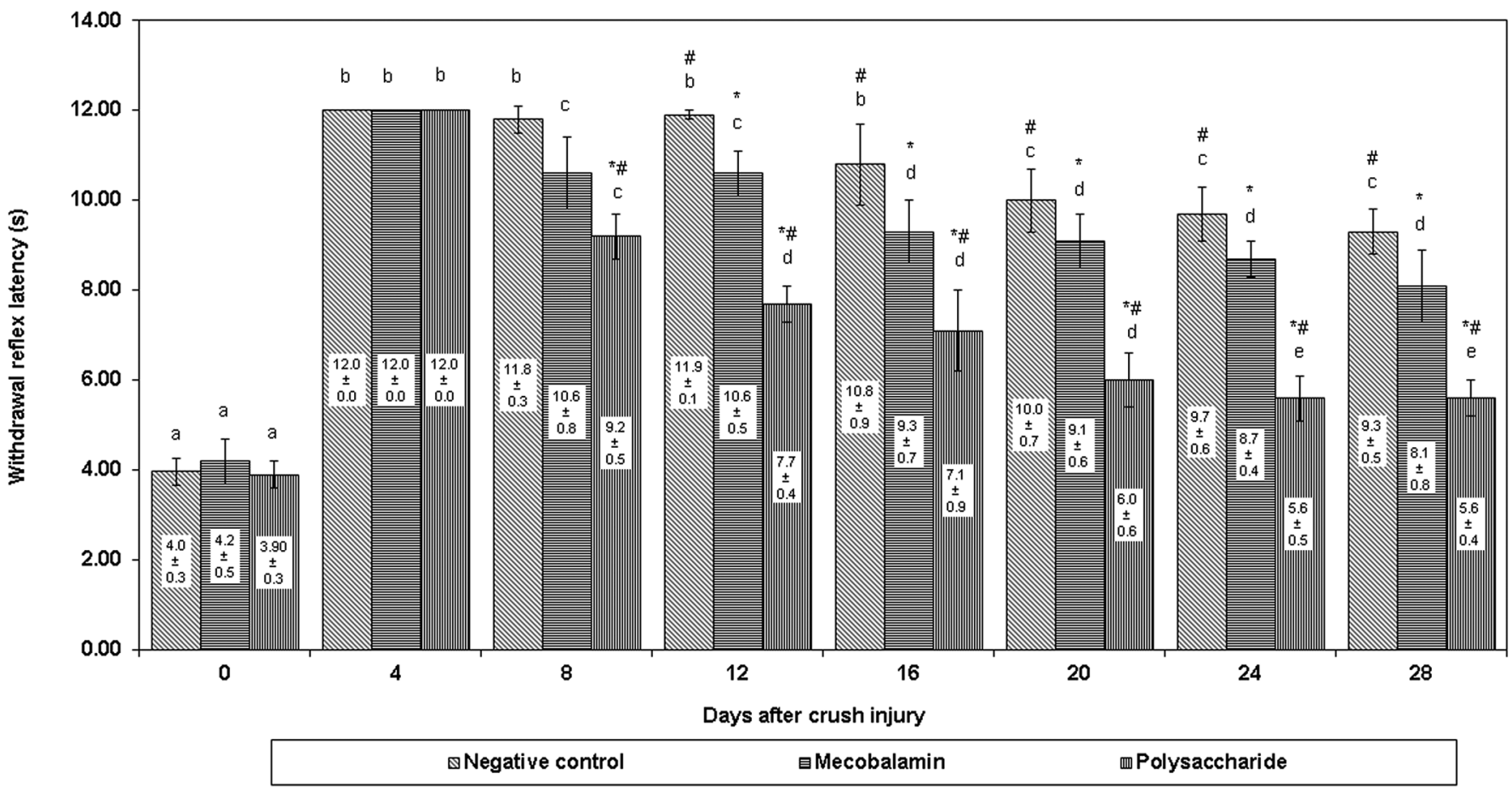

Figure 2. Mean withdrawal reflex latency (WRL) for right hind limb induced by thermal stimulation. Values in seconds (s) were obtained by performing WRL test to evaluate the nociceptive function. Each bar represents WRL from 6 animals per group. Asterisks $\left({ }^{*}\right)$ indicate significant differences $(P<0.05$, DMRT) in values for different groups compared to the negative control within a same experimental day. Hash signs $(\#)$ indicate significant differences $(P<0.05$, DMRT) in values for different groups compared to the positive control within a same experimental day. The same alphabet indicates no significant differences and different alphabets indicate significant differences $(P<0.05$, ANOVA $)$ between different experimental days in the same group. 
Figure 3 shows the in vivo expression of Akt within the DRG after crush injury. Double-labeled immunofluorescence demonstrated no co-localization of Akt and NF-200 in large DRG neurons. The population of large neurons is defined by the expression of NF-200 as green fluorescence. Akt staining as orange fluorescence was targeted to small neurons. Immunoreactivity for Akt was not detected in small neurons of contralateral DRG from uninjured nerve (Figure 3a). Immunoreactivity of ipsilateral
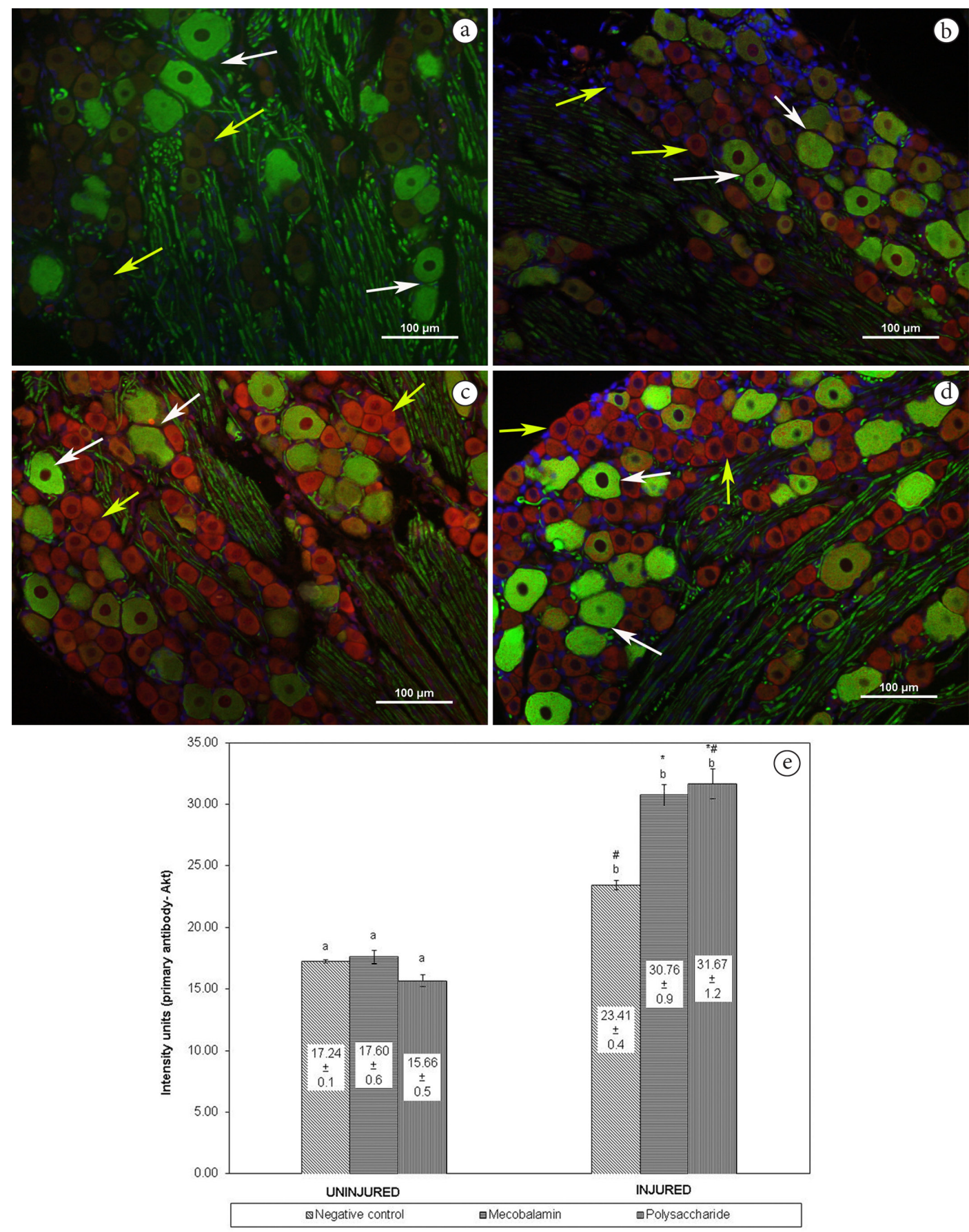

Figure 3. Akt activation in DRG neurons after crush injury. Double immunofluorescence staining between Akt (orange) and NF-200 (green) in contralateral and ipsilateral DRG. DNA was stained as blue fluorescence. (a) DRG from uninjured nerve (contralateral side); (b) DRG from injured nerve in negative control group; (c) DRG from injured nerve in positive control group; (d) DRG from injured nerve in polysaccharide group. Akt did not co-localize with large neurons. White arrows indicate large neurons whereas yellow arrows indicate small neurons. Scale bar $=100 \mu \mathrm{m}$; (e) Akt levels in the DRG as measured by intensity of immunoreactivity. Each bar represents 24 sections of DRG from 6 animals per group. Asterisks $\left(^{*}\right)$ indicate significant differences $(P<0.05, \mathrm{DMRT})$ in values for different groups compared to the negative control within a same uninjured/injured category. Hash signs $(\#)$ indicate significant differences $(P<0.05, \mathrm{DMRT})$ in values for different groups compared to the positive control within a same uninjured/injured category. The same alphabet indicates no significant differences and different alphabets indicate significant differences $(P<0.05$, ANOVA) between different categories in the same group. 
DRG in negative control (Figure $3 \mathrm{~b}$ ) and mecobalamin group (Figure 3c) were less than that observed in polysaccharide group (Figure 3d). There was a bright immunofluorescence for Akt in small neurons of ipsilateral DRG from injured nerve in polysaccharide group. Intensity measurement of Akt immunoreactivity in DRG was shown in Figure 3e. In ipsilateral DRG, the intensity recorded followed the order polysaccharide
$(31.67 \pm 1.2)>$ mecobalamin $(30.76 \pm 0.9)>$ negative control $(23.41 \pm 0.4)$. The significant increase in intensity was detected in polysaccharide group compared to negative control and mecobalamin groups $(P<0.05, \mathrm{n}=6)$.

Figure 4 shows the expression of p38 MAPK within the DRG after crush injury. Double staining of p38 MAPK with NF-200 showed no co-localization in the large DRG neurons.
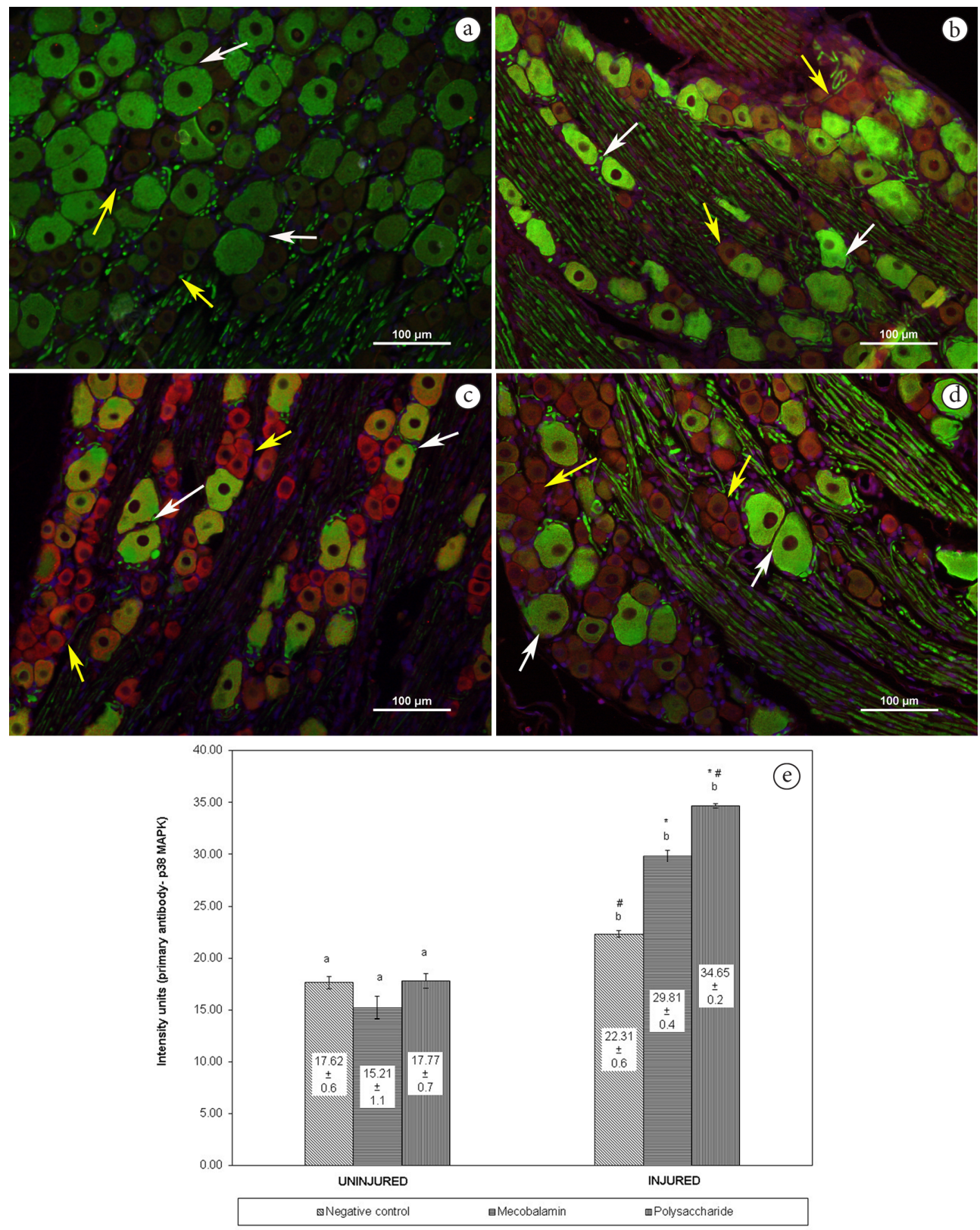

Figure 4. p38 MAPK activation in DRG neurons after crush injury. Double immunofluorescence staining between p38 MAPK (orange) and NF-200 (green) in contralateral and ipsilateral DRG. DNA was stained as blue fluorescence. (a) DRG from uninjured nerve (contralateral side); (b) DRG from injured nerve in negative control group; (c) DRG from injured nerve in positive control group; (d) DRG from injured nerve in polysaccharide group. p38 MAPK did not co-localize with large neurons. White arrows indicate large neurons whereas yellow arrows indicate small neurons. Scale bar $=100 \mu \mathrm{m}$; (e) p38 MAPK levels in the DRG as measured by intensity of immunoreactivity. Each bar represents 24 sections of DRG from 6 animals per group. Asterisks $\left(^{*}\right)$ indicate significant differences $(P<0.05$, DMRT) in values for different groups compared to the negative control within a same uninjured/injured category. Hash signs $(\#)$ indicate significant differences $(P<0.05, D M R T)$ in values for different groups compared to the positive control within a same uninjured/injured category. The same alphabet indicates no significant differences and different alphabets indicate significant differences $(P<0.05$, ANOVA) between different categories in the same group. 
Immunoreactivity for p38 MAPK was not detected in neurons of contralateral DRG (Figure 4a). In ipsilateral DRG, the intensity recorded followed the order polysaccharide $(34.65 \pm 0.2)>$ mecobalamin $(29.81 \pm 0.4)>$ negative control $(22.31 \pm 0.6)$ (Figure 4e).

Although all small neurons were stained brightly with p38 MAPK (Figure $4 \mathrm{~b}-4 \mathrm{~d}$ ), higher level of the antigen was detected in ipsilateral DRG of rats treated with polysaccharide compared to negative control and mecobalamin $(P<0.05, \mathrm{n}=6)$.

Rat endothelial cell antigen (RECA-1) positive-cells delineated the tube-shaped blood vessels in contralateral uninjured nerve (Figure 5a). Immunoreactivity for RECA-1 decreased in negative control group after crush injury (Figure 5b). However, there was a bright immunofluorescence for the antigen in the
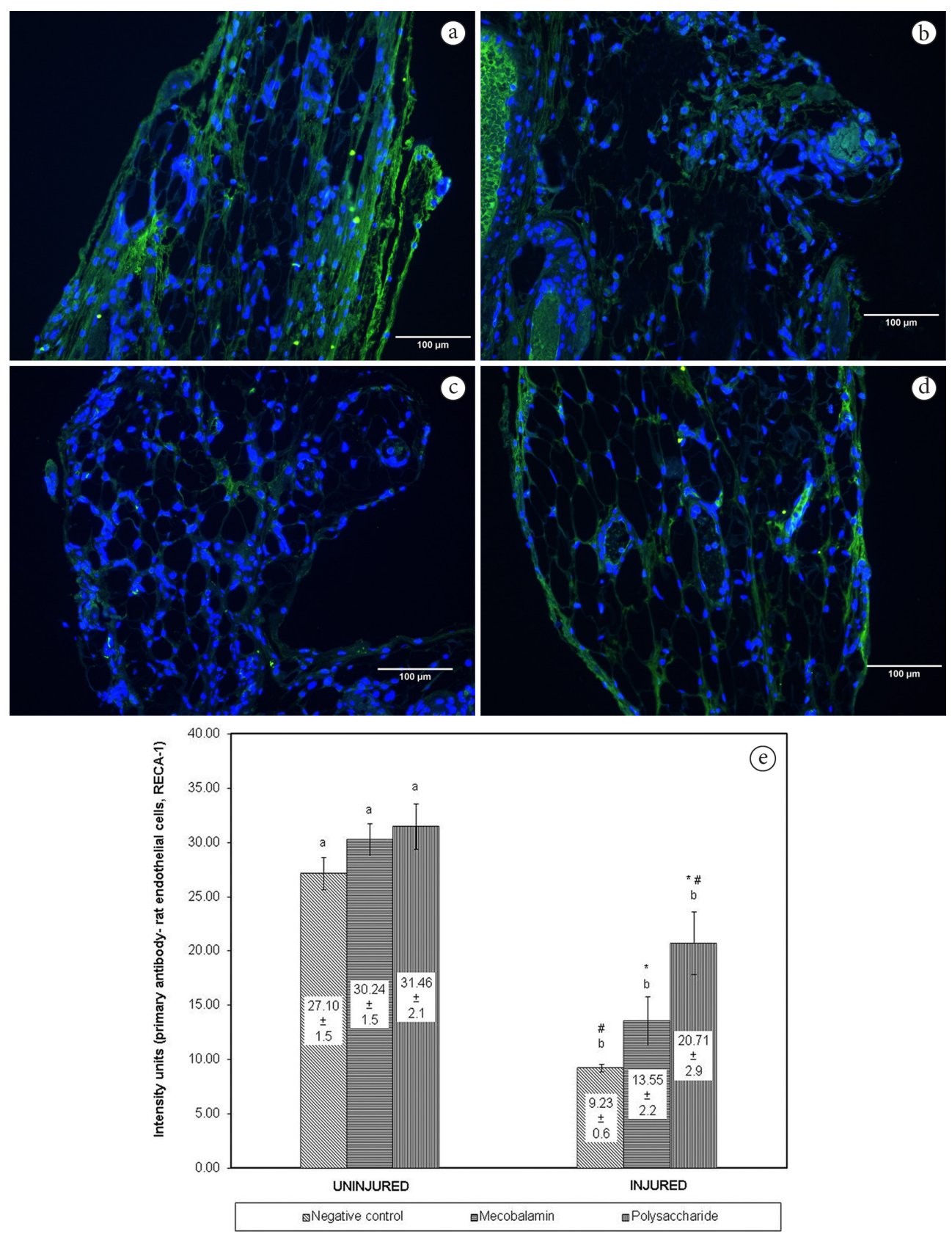

Figure 5. Distribution of rat endothelial cells antigen-1 (RECA-1)-positive microvessels in the peroneal nerve. Fluorescence imaging staining for RECA-1 as green fluorescent lines. DNA was stained as blue fluorescence. (a) Uninjured nerve (contralateral side); (b) Distal to the injury site of injured nerve in negative control group; (c) Distal to the injury site of injured nerve in positive control group; (d) Distal to the injury site of injured nerve in polysaccharide group. Scale bar $=100 \mu \mathrm{m}$; (e) RECA-1 levels in the peroneal nerve as measured by intensity of immunoreactivity. Each bar represents 24 sections of DRG from 6 animals per group. Asterisks $\left(^{*}\right)$ indicate significant differences $(P<0.05, \mathrm{DMRT})$ in values for different groups compared to the negative control within a same uninjured/injured category. Hash signs $(\#)$ indicate significant differences $(P<0.05$, DMRT) in values for different groups compared to the positive control within a same uninjured/injured category. The same alphabet indicates no significant differences and different alphabets indicate significant differences $(P<0.05$, ANOVA) between different categories in the same group. 
regenerating axons distal to the injury site in the treated groups (Figure $5 \mathrm{c}$ and $5 \mathrm{~d}$ ). Blood vessels regained their tube-shape in the treated groups at the same time point. Expression of RECA-1 occurred in regenerating axons and upregulated by polysaccharide from $H$. erinaceus after crush injury. Figure $5 \mathrm{e}$ shows the intensity measurement of RECA-1 immunoreactivity in the peroneal nerve axon. In injured nerve, the intensity followed the order polysaccharide $(20.71 \pm 2.9)>$ mecobalamin $(13.55 \pm 2.2)>$ negative control $(9.23 \pm 0.6)$. Intensity of RECA-1 was significantly higher $(P<0.05)$ in the polysaccharide and mecobalamin groups compared to negative control group.

\section{Discussion}

Natural products have been traditionally accepted as remedies due to popular belief that they present minor adverse effects. In our study, pre-treatment with polysaccharides was employed to build up strength and immune system before peripheral nerve injury. Studies on crush injury models in peripheral nerves have shown better regeneration and functional recovery when therapies were directed against ischemia-reperfusion injury by antioxidants (Algora et al., 1996). With this in mind, the present study evaluated the peripheral nerve regeneration activity of $H$. erinaceus that has been shown to possess antioxidant activity (Wong et al., 2009b) as an alternative herbal pharmacotherapy for nerve repair.

Thermal hyperalgesia associated with crush-induced neuropathy are flanked by peripheral and central events, during which infiltration of non-neuronal cells, such as Schwann cells, mast cells, macrophages and astroglia, play a determinant role (Marchand et al., 2005; Mika et al., 2008; Scholz \& Woolf, 2007). These elements release factors (TNFa, prostaglandins, NGF) that initiate and maintain sensory anomalies after injury. They act on the axons or being retrogradely transported to cell bodies in the DRG where they can modify the gene expression (Scholz \& Woolf, 2007).

Polysaccharide from $H$. erinaceus could attenuate thermal hyperalgesia induced by peroneal nerve injury and enhanced the reflex response of the right hind limb after 8 days of injury and these significant changes were observed subsequently during the remainder of the time period. This effect is due to faithful reinnervation of sensory receptors by their original axons. Even when good motor recovery occurs, sensory deficits, particularly in proprioception, may impair the functional outcome. Sensory recovery may continue for a longer period of time than motor recovery as the axons of sensory neurons provide cutaneous sensation to a larger area than it had prior to the injury (Harden et al., 2012). We have shown that initiation of motor functional recovery began after 4 days of injury in the $H$. erinaceus-treated rats (Wong et al., 2011) which is earlier than the sensory functional recovery in this study.

Further, this is the first study that reveals the connection between activation of Akt and p38 MAPK signaling pathways and restoration of BNB integrity with sensory functional recovery following crush injury by a medicinal mushroom renowned for its neuroprotective effects. Upregulation of Akt and p38 MAPK after 12 days of crush injury is consistent with the beginning of sensory functional recovery of injured right hind limb.

Injury to neurons results in complex sequences of molecular responses that play an important role in the successful regenerative response and the eventual recovery of sensory function. Peripheral sensitization causes activation of Akt, and p38 MAPK in small DRG neurons. These contributed to pain hypersensitivity found at the site of tissue damage and inflammation. In general, DRG neurons can be divided into large $\left(>1200 \mu \mathrm{m}^{2}\right)$, medium $\left(600-1200 \mu \mathrm{m}^{2}\right)$ and small $\left(<600 \mu \mathrm{m}^{2}\right)$ neurons. Small neurons respond to thermal, mechanical and chemical nociceptive stimulations whereas large neurons transmit touch and proprioceptive sensations (Wen et al., 2009). Akt and p38 MAPK did not co-localize with NF-200 in large DRG neurons. It is possible that polysaccharide from $H$. erinaceus could trigger the expression of protein kinases that regulate nociceptive function and inflammation in the enhancement of nerve regeneration. Grothe et al. (1997) proposed that the upregulation of growth factors in satellite glial cells after injury may contribute to the recovery and regeneration processes. These supporting cells wrap completely around the sensory neurons.

Akt promotes cell survival by inhibiting apoptosis through phosphorylation and inactivation of several targets, including Bad, forkhead transcription factors, c-Raf, and caspase- 9 (Franke et al., 1997). p38 MAPK is regulated by streess-inducing signals such as osmotic stress, UV radiation, pro-inflammatory cytokines and certain toxins (Okada et al., 2007). Activation of MAPK pathway is essential for neurite outgrowth, regeneration, synaptic plasticity and memory functions in mature neurons (Sweatt, 2001).

Blood-nerve barrier is a dynamic and competent interface between the endoneurial microenvironment and the surrounding extracellular space or blood. It is localised at the innermost layer of the multilayered ensheathing perineurium and endoneurial microvessels. Anatomically, the endothelial cells of the endoneurial microvessels are linked together by tight junctions that limit the entrance of macromolecular substances (Bain et al., 1990).

Hericium erinaceus effectively restores the integrity of the $\mathrm{BNB}$ after crush injury. The breakdown and restoration of BNB is an important reaction in nerve regeneration. We suggest that the processes are associated with the changes in the expression of tight junctional proteins. Breakdown of BNB allows acceptance of cytokines and various growth factors within the endoneurial space. Once the nerve has been regenerated, BNB restores and endoneurial microvessels become impermeable.

\section{Conclusions}

We demonstrated a neuroregenerative role of polysaccharide from $H$. erinaceus fresh fruit bodies in the peripheral nervous system. The polysaccharide promotes more rapid return of sensory function of injured peripheral nerve and facilitating recovery in rats with traumatic nerve injury. Further studies are needed to understand the regulatory mechanism of the blood-nerve barrier that can lead to the development of novel methods for treatment and/or prevention of complications after traumatic injuries of peripheral nerves. We see this as a 
critical step towards further development of this mushroom in the alternative therapies and practices.

\section{Acknowledgements}

This work was supported by University of Malaya RG268-13AFR and Ministry of Higher Education Malaysia through High Impact Research Grant UM.C/625/1/HIR/MoE/SC/02. We would like to thank Mrs. Poh-Guat Cheng of Vita Agrotech for the constant supply of $H$. erinaceus.

\section{References}

Algora, J., Chen, L. E., Seaber, A. V., Wong, G. H., \& Urbaniak, J. R. (1996). Functional effects of lymphotoxin on crushed peripheral nerve. Microsurgery, 17(3), 131-135. http://dx.doi.org/10.1002/(SICI)10982752(1996)17:3<131::AID-MICR6>3.0.CO;2-P. PMid:9016456.

Bain, J. R., Hudson, A. R., Mackinnon, S. E., Gentili, F., \& Hunter, D. (1990). The blood-nerve barrier in peripheral nerve injury, repair, and regeneration. In M. Samii (Ed.), Peripheral nerve lesions (pp. 130-142). Berlin: Springer-Verlaag.

Campbell, J. N. (2001). Nerve lesions and the generation of pain. Muscle \& Nerve, 24(10), 1261-1273. http://dx.doi.org/10.1002/mus.1143. PMid:11562904.

Franke, T. F., Kaplan, D. R., \& Cantley, L. C. (1997). PI3K: downstream AKTion blocks apoptosis. Cell, 88(4), 435-437. http://dx.doi. org/10.1016/S0092-8674(00)81883-8. PMid:9038334.

Grothe, C., Meisinger, C., Hertenstein, A., Kurz, A., \& Wewetzer, K. (1997). Expression of fibroblast growth factor-2 and fibroblast growth factor receptor 1 messenger RNAs in spinal ganglia and sciatic nerve: regulation after peripheral nerve lesion. Neuroscience, 76(1), 123-135. http://dx.doi.org/10.1016/S0306-4522(96)00355-7. PMid:8971765.

Harden, R. N., Richardson, K., Shufelt, M., \& Revivo, G. (2012). Neuropathic pain: an interdisciplinary approach. In D.M. Simpson, J.C. McArthur \& R.H. Dworkin (Eds.), Neuropathic pain: mechanisms, diagnosis and treatment (pp. 472-484). New York: Oxford University Press.

Hu, D., Hu, R., \& Berde, C. B. (1997). Neurologic evaluation of infant and adult rats before and after sciatic nerve blockade. Anesthesiology, 86(4), 957-965. http://dx.doi.org/10.1097/00000542-19970400000026. PMid:9105240.

Kawagishi, H., Zhuang, C., \& Yunoki, R. (2008). Compounds for dementia from Hericium erinaceum. Drugs of the Future, 33(2), 149-155. http://dx.doi.org/10.1358/dof.2008.033.02.1173290.

Kolotushkina, E. V., Moldavan, M. G., Voronin, K. Y., \& Skibo, G. G. (2003). The influence of Hericium erinaceus extract on myelination process in vitro. Fiziologicheskii Zhurnal, 49(1), 38-45. PMid:12675022.

Lai, P. L., Naidu, M., Sabaratnam, V., Wong, K. H., David, R. P., Kuppusamy, U. R., Abdullah, N., \& Malek, S. N. (2013). Neurotrophic properties of Hericium erinaceus (Bull.: Fr.) Pers. grown in tropical climate of Malaysia. International Journal of Medicinal Mushrooms, 15(6), 539-554. http://dx.doi.org/10.1615/IntJMedMushr.v15.i6.30. PMid:24266378.

Marchand, F., Perretti, M., \& McMahon, S. B. (2005). Role of the immune system in chronic pain. Nature Reviews. Neuroscience, 6(7), 521-532. http://dx.doi.org/10.1038/nrn1700. PMid:15995723.

Mika, J., Korostynski, M., Kaminska, D., Wawrzczak-Bargiela, A., Osikowicz, M., Makuch, W., Przewlocki, R., \& Przewlocka, B. (2008). Interleukin-1 has antiallodynic and antihyperalgesic activities in a rat neuropathic pain model. Pain, 138(3), 587-597. http://dx.doi. org/10.1016/j.pain.2008.02.015. PMid:18374486.

Moldavan, M. G., Grygansky, A., Kolotushkina, O. V., Kirchhoff, B., Skibo, G. G., \& Pedarzani, P. (2007). Neurotropic and trophic action of lion's mane mushroom Hericium erinaceus (Bull.: Fr) Pers. (Aphyllophoromycetideae) extracts on nerve cells in vitro. International Journal of Medicinal Mushrooms, 9(1), 15-28. http:// dx.doi.org/10.1615/IntJMedMushr.v9.i1.30.

Okada, Y., Ueshin, Y., Isotani, A., Saito-Fujita, T., Nakashima, H., Kimura, K., Mizoguchi, A., Oh-hora, M., Mori, Y., Ogata, M., Oshima, R. G., Okabe, M., \& Ikawa, M. (2007). Complementation of placental defects and embryonic lethality by trophoblast-specific lentiviral gene transfer. Nature Biotechnology, 25(2), 233-237. http://dx.doi. org/10.1038/nbt1280. PMid:17220877.

Phan, C. W., Lee, G. S., Hong, S. L., Wong, Y. T., Brkljača, R., Urban, S., Abd Malek, S. N., \& Sabaratnam, V. (2014). Hericium erinaceus (Bull.: Fr) Pers. cultivated under tropical conditions: isolation of hericenones and demonstration of NGF-mediated neurite outgrowth in PC12 cells via MEK/ERK and PI3K-Akt signaling pathways. Food \& Function, 5(12), 3160-3169. PMid:25288148.

Roy, S. K., Maiti, D., Mondal, S., Das, D., \& Islam, S. S. (2008). Structural analysis of a polysaccharide isolated from the aqueous extract of an edible mushroom, Pleurotus sajor-caju, cultivar Black Japan. Carbohydrate Research, 343(6), 1108-1113. http://dx.doi.org/10.1016/j. carres.2008.02.015. PMid:18336803.

Scholz, J., \& Woolf, C. J. (2007). The neuropathic pain triad: neurons, immune cells and glia. Nature Neuroscience, 10(11), 1361-1368. http://dx.doi.org/10.1038/nn1992. PMid:17965656.

Sweatt, J. D. (2001). The neuronal MAP kinase cascade: a biochemical signal integration system subserving synaptic plasticity and memory. Journal of Neurochemistry, 76(1), 1-10. http://dx.doi.org/10.1046/ j.1471-4159.2001.00054.x. PMid:11145972.

Wen, Y. R., Suter, M. R., Ji, R. R., Yeh, G. C., Wu, Y. S., Wang, K. C., Kohno, T., Sun, W. Z., \& Wang, C. C. (2009). Activation of p38 mitogen-activated protein kinase in spinal microglia contributes to incision-induced mechanical allodynia. Anesthesiology, 110(1), 155-165. http://dx.doi.org/10.1097/ALN.0b013e318190bc16. PMid:19104183.

Wong, K. H., Kanagasabapathy, G., Naidu, M., David, P., \& Sabaratnam, V. (2014). Hericium erinaceus (Bull.: Fr.) Pers., a medicinal mushroom, activates peripheral nerve regeneration. Chinese Journal of Integrative Medicine. http://dx.doi.org/10.1007/s11655-014-16242. PMid:25159861.

Wong, K. H., Naidu, M., David, P., Abdulla, M. A., Abdullah, N., Kuppusamy, U. R., \& Sabaratnam, S. (2011). Peripheral nerve regeneration following crush injury to rat peroneal nerve by aqueous extract of medicinal mushroom Hericium erinaceus (Bull.: Fr) Pers. (Aphyllophoromycetideae). Evidence-based Complementary and Alternative Medicine, 2011, 580752. http://dx.doi.org/10.1093/ecam/ neq062. PMid:21941586.

Wong, K. H., Naidu, M., David, R. P., Abdulla, M. A., Abdullah, N., Kuppusamy, U. R., \& Sabaratnam, S. (2009a). Functional recovery enhancement following Injury to rodent peroneal nerve by lion's mane mushroom, Hericium erinaceus (Bull.: Fr.) Pers. (Aphyllophoromycetideae). International Journal of Medicinal Mushrooms, 11(3), 225-236. http:// dx.doi.org/10.1615/IntJMedMushr.v11.i3.20.

Wong, K. H., Naidu, M., David, R. P., Bakar, R., \& Sabaratnam, V. (2012). Neuroregenerative potential of lion's mane mushroom, Hericium erinaceus (Bull.: Fr.) Pers. (higher basidiomycetes), in the treatment of peripheral nerve injury. International Journal of 
Medicinal Mushrooms, 14(5), 427-446. http://dx.doi.org/10.1615/ IntJMedMushr.v14.i5.10. PMid:23510212.

Wong, K. H., Sabaratnam, V., Abdullah, N., Kuppusamy, U. R., \& Naidu, M. (2009b). Effects of cultivation techniques and processing on antimicrobial and antioxidant activities of Hericium erinaceus (Bull.: Fr.) Pers. extracts. Food Technology and Biotechnology, 47(1), 47-55.
Wong, K.-H., Vikineswary, S., Abdullah, N., Naidu, M., \& Keynes, R. (2007). Activity of aqueous extracts of lion's mane mushroom Hericium erinaceus (Bull.: Fr.) Pers. (Aphyllophoromycetideae) on the neural cell line NG108-15. International Journal of Medicinal Mushrooms, 9(1), 57-65. http://dx.doi.org/10.1615/IntJMedMushr. v9.i1.70. 